\title{
1 Confocal Raman Microscopy of Frozen Bread Dough
}

2 Authors:

3 Julien Huen ${ }^{a, *}$, Christian Weikusat ${ }^{b}$, Maddalena Bayer-Giraldi $^{\mathrm{b}}$, Ilka Weikusat ${ }^{\mathrm{b}}$, Linda Ringer ${ }^{\mathrm{a}}$,

$4 \quad$ Klaus Lösche ${ }^{a}$

$5 \quad{ }^{a}$ ttz Bremerhaven, BILB-EIBT, Am Lunedeich 12, 27572 Bremerhaven, Germany

$6{ }^{b}$ Alfred Wegener Institute for Polar and Marine Research, Am Alten Hafen 26, 27570 Bremerhaven,

7 Germany

$8 \quad *$ Corresponding author. Tel.: +49 471 80934-241; fax: +49 471 80934-299.

9 E-mail addresses: jhuen@ttz-bremerhaven.de (Julien Huen), christian.weikusat@awi.de (Christian

10 Weikusat), maddalena.bayer@awi.de (Maddalena Bayer-Giraldi), ilka.weikusat@awi.de (Ilka

11 Weikusat), Iringer@ttz-bremerhaven.de (Linda Ringer), kloesche@ttz-bremerhaven.de (Klaus Lösche)

12 Keywords: Confocal Raman Microscopy, Frozen Bread Dough, Microstructure, Ice crystals. 


\section{Abstract}

14 The use of freezing technology is well established in industrial and craft bakeries and is still gaining

15 importance. In order to optimize recipes and processes of frozen baked goods, it is essential to be able to investigate the products' microstructure. Especially ice crystals and their interaction with the other components of the frozen products are of interest. In this study, frozen wheat bread dough was investigated by confocal Raman microscopy. The Raman spectra measured within the dough were compared with spectra of the main components of frozen dough, i.e. ice, liquid water, starch, gluten and yeast. In this way, the spatial distribution of the single components within the dough was determined and corresponding images of the frozen dough microstructure were generated. On these images, ice appears as a continuous network rather than as isolated crystals. We suggest that this method may be appropriate for characterizing crystallization phenomena in frozen baked goods, allowing to better understand the reasons for quality losses and to develop strategies for avoiding such losses. 


\section{Introduction}

As the bakery business is being concentrated and rationalized, increasing use is made of freezing technology in production and distribution (Le Bail et al., 2012). Freezing allows a separation in time and space of process operations that would traditionally be performed in one run and in one place.

In bread-making, freezing is used at several stages of production: for non-fermented or partlyfermented dough, for partly or fully baked products (Le Bail et al., 2012). Depending on the application, the products are kept frozen for a few hours or for several weeks or months. A large variety of equipment, including shock-freezers, fermentation interrupters, climatic chambers, and cold storage rooms are used for realizing the operations of freezing, cold storage and thawing.

Although the intention when using freezing is to keep the product in a steady state, in practice a number of physical and chemical phenomena occur, affecting the quality of the final product in a mostly negative way. Among these phenomena, the formation of ice crystals is believed to be of primary importance for two main reasons (Berglund et al. 1991, Baier-Schenk et al. 2005a):

(1) Ice crystals are made of pure water which is being separated from the product matrix.

Cryoconcentration occurs in the liquid phase, which may influence the solubility of proteins and the activity of enzymes. During storage, ice crystals grow due to recrystallization, especially in the pores, thus further modifying the distribution of water in the product. (2) Ice crystals may mechanically damage the dough components, especially the gluten network and the yeast cells, because the freezing front exerts stress on the surrounding material. This effect is believed to be more pronounced as the crystal size increases due to recrystallization.

In order to optimize the recipes and the production processes of frozen baked goods, it is essential to be able to monitor the phenomena occurring in the products in the frozen state. Differential scanning calorimetry (DSC) allows quantitative investigations of ice crystallization. For monitoring the size and the distribution of the ice crystals as well as their mechanical interactions with the other components of the dough, imaging techniques are required. So far, scanning electron microscopy in 
the frozen state (cryo SEM, Zounis et al. 2002, Esselink et al., 2003, Baier-Schenk et al. 2005a) and confocal laser scanning microscopy (CLSM, Baier-Schenk et al. 2005b) have been used for that purpose. Cryo SEM has allowed demonstrating the growth of ice crystals within the pores over storage time and CLSM to identify regions of preferential nucleation. However, in both techniques, a difficulty is the limited possibility to unambiguously differentiate the ice crystals from the other components of the dough. In cryo SEM, this differentiation is performed based on the regular shape of the crystals - but this is only valid in the pores, where ice crystals can grow without spatial constraints. In CLSM, changes in the reflection properties were attributed to ice crystal growth. However, this method did not allow for generating precise images of the ice crystal structure. Due to these limitations, little is known about the structure of the ice crystals that are entrapped in the dough matrix, which yet represent the main part of the frozen water.

Raman spectroscopy belongs to the group of vibrational spectroscopies (Smith et al., 2005). It utilizes the inelastic scattering of light photons on molecules or molecular groups, called Raman effect. If the molecule (or group) has suitable vibrational modes, a photon can transfer a fraction of its energy to the vibration (Stokes scattering). The positions of the Raman bands directly give the energy of the detected vibrations. The ensemble of Raman active vibrations is characteristic for each compound and can range from single bands to very complex multi-band spectra. Raman spectroscopy is a nondestructive method requiring very little sample preparation and it is suitable for a wide range of materials. If high-quality reference spectra are available, it is a very sensitive tool for phase identification.

With the implementation of Raman spectroscopy in confocal microscopy in the late 1990s, it became possible to use Raman data for microimaging purposes. Applications were developed in a variety of scientific fields including mineralogy, petrography, polymer science, pharmaceutical research (Dieing et al., 2011), biomedical diagnostics (Krafft et al., 2012) and glaciology (Weikusat et al., 2012). In agricultural and food science and more specifically in cereal science, only little use has been made of this technique so far. Piot et al. $(2000,2001,2002)$ used confocal Raman microscopy for exploring 
the spatial distribution of starch, gluten, arabinoxylan and ferulic acid in wheat grains. Recently, Jääskeläinen et al. (2013) performed similar investigations with higher (sub- $\mu \mathrm{m})$ spatial resolution on barley and wheat grains.

Based on the fact that confocal Raman microscopy has shown to be suitable for characterising both ice crystals and the main components of cereals, our objective was to develop a measurement method appropriate for investigating the microstructure of frozen bread dough.

\section{Experimental}

\subsection{Raw materials and equipment}

The following ingredients were used in the experiments: Wheat flour type 550 (Roland Mühle, Germany), compressed yeast (Frischhefe, Deutsche Hefewerke GmbH, Germany), and salt (Suprasel fine, Suprasel, The Netherlands).

Raman measurements were performed on a WITec Alpha 300R microspectroscopy system equipped with a frequency-doubled Nd:YAG laser $(\lambda=532 \mathrm{~nm}$ ), an UHTS300 Raman Spectrometer (grating: 600 grooves/mm, pixel resolution $<0.09 \mathrm{~nm}$ ) with a Peltier-cooled DV401A-BV CCD detector (peak quantum efficiency at $\sim 550 \mathrm{~nm}$ and $-60^{\circ} \mathrm{C}$ : $\left.>95 \%\right)$ and a 50x LWD objective, operated in a cold laboratory at $-15^{\circ} \mathrm{C}$ at the Alfred-Wegener Institute. The laser power on the sample was $<30 \mathrm{~mW}$.

\subsection{Assessment of Raman spectra of single dough components}

The Raman spectra of ice, liquid water, starch, gluten, and yeast were assessed using the following procedure.

\subsubsection{Sample preparation}

A 3.5\% (w/v) salt solution in bidistilled water was prepared. One droplet of this solution $(20 \mu \mathrm{L})$ was placed on a microscope slide, covered with a cover slip using a $2 \mathrm{~mm}$ spacer to standardize thickness, and frozen at $-20^{\circ} \mathrm{C}$. In this way ice crystals and a liquid phase (cryoconcentrated salt solution) were 
formed. The salt present in the liquid phase is expected to influence the Raman spectrum only to a minimal extent, as its main component $\mathrm{NaCl}$ ( $\geq 99,8 \%$ according to the supplier's specification) has no molecular vibration.

Wheat flour was hydrated and separated into a starch suspension and a wet gluten piece using a Glutomatic 2200 from Perten Instruments, Sweden. One droplet of the starch suspension was placed on a microscope slide, covered with a cover slip using a $2 \mathrm{~mm}$ spacer and frozen at $-20^{\circ} \mathrm{C}$. The same was done with a small portion of the wet gluten piece and of the compressed yeast block.

\subsubsection{Measurement}

The Raman spectrum of each of the samples representative for the individual dough components was measured at 10 different points, with 20 accumulations of $1 \mathrm{~s}$ each per point, and the average spectrum was calculated for each component.

\subsection{Dough sample preparation}

Three frozen dough samples were prepared at three different days in the following way: $50 \mathrm{~g}$ of wheat flour, $28 \mathrm{~g}$ of bidistilled water, $1.5 \mathrm{~g}$ of compressed yeast and $1 \mathrm{~g}$ of salt were mixed and kneaded to a dough in a Brabender Farinograph AT at $20^{\circ} \mathrm{C}$. The mixing time was 2 minutes at $36 \mathrm{rpm}$ and the kneading time $4 \mathrm{~min}$ at $63 \mathrm{rpm}$. After kneading, the dough was allowed to rest for $15 \mathrm{~min}$ at room temperature. Subsequently, a small piece (approx. $250 \mathrm{mg}$ ) of the inner part of the dough was cut out, placed on a microscope slide, covered with a cover slip using a $2 \mathrm{~mm}$ spacer and frozen at $-20^{\circ} \mathrm{C}$.

\subsection{Confocal Raman microscopy of frozen dough samples}

On the day following preparation, the samples were transferred to the microscopy laboratory at $-15^{\circ} \mathrm{C}$. Before measurement, the samples were kept for at least one hour at $-15^{\circ} \mathrm{C}$ to stabilize at that temperature. 
123 For each of the 3 frozen dough samples, an area of $100 \times 100 \mu \mathrm{m}$ was measured with a resolution of $124200 \times 200$ points and an integration time of $1 \mathrm{~s}$ per point, resulting in a measurement time of approx. 12512 hours.

\subsection{Confocal Raman microscopy: data processing and imaging}

127 The data from the area scans were processed in two different ways to produce images showing the spatial distribution of the single dough components (ice, liquid water, starch, gluten and yeast).

In the first method, single Raman bands characteristic for each component were integrated.

Monochrome images were generated representing the intensity of the individual bands at each measurement point. The spectral ranges of the chosen bands are given in Table 1 and are marked in blue in Figure 1.

The second method considered the full Raman spectra instead of single bands. In that method, the Raman spectrum measured at each point of the sample was assumed to be a linear combination of the spectra of the single dough components. After performing a $3^{\text {rd }}$ order polynomial background subtraction on all spectra, a multiple linear regression was completed using the function Basis Analysis of the WITec Project software (release 2.10, WITec GmbH, Ulm, Germany). The assessed regression coefficients were used as indicators of the concentration of the individual dough components, and corresponding monochrome images were generated. This method is well established in confocal Raman microscopy and was used among others by Jääskeläinen et al. (2013).

141 The monochromatic images showing the distribution of the single components were combined to colour images in which each colour represents one component. This allows visualizing the position and distribution of the components relative to each other. 


\subsection{Raman spectra of single dough components}

The measured spectra of the single dough components are presented in Figure 1, and bands of particular interest are listed in Table 1.

Ice is especially characterized by the $\mathrm{OH}$ stretching band with a maximum in the spectral range of 3080-3200 $\mathrm{cm}^{-1}$, as described by Đuričković et al. (2011). This band was not found in the spectra of the other dough components. The spectrum of liquid water is dominated by the $\mathrm{OH}$ stretching band with a maximum in the range of $3300-3420 \mathrm{~cm}^{-1}$, and also embodies the $\mathrm{OH}$ bending band (1580$\left.1640 \mathrm{~cm}^{-1}\right)$. Starch shows a series of bands, reflecting its molecular complexity. These bands were already reported by Piot et al. (2000) and Fechner et al. (2005) and their assignment discussed by these authors. The $\mathrm{CH}$ stretching band $\left(2800-3050 \mathrm{~cm}^{-1}\right)$ and the $\mathrm{OH}$ stretching band are both strongly represented. The narrow band in the range of $460-510 \mathrm{~cm}^{-1}$, which is attributed to the stretching vibration of the carbon network of starch, was not found in spectra of the other dough components. The gluten spectrum shows a higher base signal, due to fluorescence, and a series of bands that were described and which assignment was discussed by Piot et al. (2000). As in starch, the $\mathrm{CH}$ stretching band is strongly represented. The band in the range of $1645-1690 \mathrm{~cm}^{-1}$ is attributable, at least partly, to amide I (see "band position and assignment" in the discussion). measurements of Rösch et al. (2006) with Saccharomyces cerevisiae. In the range of 1645-1690 $\mathrm{cm}^{-1}$, yeast show a signal similar to gluten, yet with lower intensity. The band in the range of $740-766 \mathrm{~cm}^{-1}$, which was assigned by the latter authors to tryptophan, appears to be specific for yeast in the studied dough system in terms of intensity - gluten and to a lesser extent starch also have bands in this spectral range, but they are weaker. 
168

169

170

171

172

173

174

175

The figures 2, 3 and 4 show the spatial distribution of the individual dough components within the three samples, as determined by both data processing methods (band integration and multiple linear regression). The distribution of liquid water, however, was determined only by multiple linear regression, as the $\mathrm{OH}$ Raman bands of liquid water overlap with the $\mathrm{OH}$ bands of the starch and the gluten spectra - in other words, liquid water has no specific single Raman band in the frozen dough system. Multiple linear regression, on the other hand, failed to allow for a determination of the distribution of yeast, as is discussed below. For each sample, a colour image showing the relative spatial distribution of the single dough components was generated.

Both representations are complementary. The monochrome images give more details about the structure of the single components. Due to the depth of field of a few $\mu \mathrm{m}$, it gives some insights into the 3-dimensional structure. Elements located a few $\mu \mathrm{m}$ above or below the focal plane are still being detected but lead to a weaker signal, which is represented by a lower pixel brightness. The colour images, on the other hand, show how the dough components are spatially organized relatively to each other in the focal plane.

Starch appears on the pictures as large granules with a diameter of $20-25 \mu \mathrm{m}$ and smaller granules with a diameter of 2-5 $\mu \mathrm{m}$. Gluten appears as fibrils organised around the starch granules, partly with a spatial orientation as parallel strands. In the three samples studied, ice appears as a continuous network rather than as single crystals. This network structure is better visible on the single-phase than on the multi-phase pictures, due to the higher depth of field. Small ice blocks with a diameter of 1-10 $\mu \mathrm{m}$, integrated in the ice network, are observed in some of the spaces between the starch granules. Yeast cells appear on the picture as ellipsoids with a size of 4-5 $\mu \mathrm{m}$, homogeneously distributed within the samples. The yeast images also show a background noise, especially in the gluten-rich regions. Liquid water appears to be present in the areas where no other phase is present. 


\section{Sample integrity}

193

In Raman microscopy, in order to obtain a detectable signal, a laser beam with high power density needs to be applied in the focus area, which can result in heating and structural alteration. Especially with frozen samples it is therefore essential to ascertain the integrity of the sample after the measurements. In the case of the samples of the present study, routine microscopic inspection of the Raman-mapped sample areas showed no signs of damage. Although it is not possible to measure temperature within the sample during measurement, two observations suggest that temperature was not significantly increased: (1) Ice and liquid water were found to be both strongly represented in the investigated samples; this is consistent with the DSC measurements of frozen dough by BaierSchenk et al. (2005a), which show at $-15^{\circ} \mathrm{C}$ about $50 \%$ of the water are in the frozen state, whereas the other $50 \%$ are in the liquid form; (2) Repeated mappings of the same areas yielded identical results; under the assumption of melting and recrystallization, a different distribution of ice would have been observed.

\section{Band position and assignment}

The spectral bands used for imaging in the first method were chosen both by (1) comparing the spectra of the single components on Figure 1 and searching for bands that are unique for each component and (2) using knowledge from literature on band assignment. In the case the $\mathrm{OH}$ stretching band of ice and the stretching vibration of the carbon network of starch, the high intensity of the bands and their characteristic shape allows for a clear assignment. These bands are very appropriate for identifying ice and starch in the frozen dough. The assignment of the amide I band is more complicated due to the fact that the shape of the band and the position of its maximum depends on the secondary and tertiary structure of the proteins (Tuma, 2005). A further difficulty lies in the proximity of other bands. Finally, fluorescence, which is dependent on the excitation wavelength, overlaps with the Raman signal. For these reasons, there is no certitude that the spectral 
range selected (1645-1690 $\left.\mathrm{cm}^{-1}\right)$ corresponds exclusively to amide I in gluten. In addition, it must be noted that amide I can only be seen as an imperfect indicator of gluten in the frozen dough system, as amide I signal is also expected to arise from non-gluten wheat protein and from yeast protein.

The use of the band $740-766 \mathrm{~cm}^{-1}$ for yeast identification in the dough must be considered as an empirical approach. It is unclear whether the signal measured in this range is solely attributable to the ring breathing vibration of tryptophan, nor whether tryptophan can be considered as a reliable indicator or yeast in the dough system.

\section{Unambiguous identification and imaging of the single dough components}

As discussed above, starch and ice have good single band indicators in the frozen dough system, and it is not surprising that for these components both data processing methods lead to similar pictures. The pictures generated by multiple linear regression show less noise and are therefore sharper, probably due to the fact that they are based on a broader data basis. In the case of gluten, a good match between the pictures obtained from both data processing methods is observed as well.

The identification and imaging of yeast has a lower level of confidence, due to the limitations described above. The noise observed on the images can be explained by the fact that gluten and starch also have weak bands in the chosen spectral range. Imaging of yeast using multiple linear regression was not successful, as the generated images were obviously wrong (no cell shape); this is probably due to the overlap of the yeast signals with signals from the other dough components in most spectral areas, as well as to the low abundance of yeast in the system.

Liquid water can be identified only by multiple linear regression, due to the overlap with the $\mathrm{OH}$ bands of the other components.

\section{Spatial distribution of the single dough components}

The observed spatial distribution of starch, gluten and yeast is consistent with data from literature. A bimodal size distribution of starch granules in wheat flour was reported by numerous authors like 
Stoddard et al. (1999). The observed relative distribution of starch and gluten, with gluten fibrils organised as a network around the starch granules, is consistent with observations made by other techniques like scanning electron microscopy (Yi et al., 2009), confocal scanning laser microscopy and epifluorescence light microscopy (Peighambardoust et al., 2010). The observed size and shape of the yeast cells is in line with literature data (Smith et al., 2000).

The most interesting, and really novel aspect, is the distribution of ice. The structure of ice as a continuous phase (crystal network) within the frozen dough has not, to our knowledge, been reported elsewhere so far. This continuous structure may be of importance for understanding damage to the other dough components, especially to gluten which also has a network structure meaning that in the frozen state, the gluten network and the ice crystal network coexist and are embedded in one another.

\section{Conclusions}

In our investigations, confocal Raman microscopy allowed a reliable identification and imaging of starch, ice and gluten; yeast and liquid water were identified with a lower degree of confidence. The method is non-destructive and does not require any staining. band) allows visualising the structure of ice within the frozen dough matrix. The structure of ice as a network rather than isolated crystals represents a new finding that helps understanding the interactions between the dough components in the frozen state.

We suggest that the technique described in this paper may be useful to study the influence of different freezing and storage conditions, of different storage times, and of specific ingredients such as ice structuring proteins, on the ice network structure in frozen dough. Such investigations may be conducted either on a model system like in this study (dough frozen on a microscope slide), or on microtome sections of real-life frozen products. 
The technique in itself may be refined in terms of spatial resolution by the use of an objective with a higher magnification and in terms of measurement speed by the use of a more sensitive spectrometer. The use of a different excitation wavelength could help reducing fluorescence. More detailed Raman spectroscopic studies of the single components of the dough, especially starch and gluten, may allow differentiating between sub-components such as amylose, amylopectin, gliadin and glutenin, ultimately leading to more detailed images.

Numerous further applications of cryo Raman microscopy are conceivable with other kinds of frozen foods or frozen biological samples.

\section{Acknowledgements}

This research project was supported by the German Ministry of Economics and Technology (via AiF) and the FEl (Forschungskreis der Ernährungsindustrie e.V., Bonn). Project AiF 17181 N.

Access to the cryo Raman microscope was provided by the Young Investigator Group VH-NG-802 of the Helmholtz Association.

Baier-Schenk, A., Handschin, S., Conde-Petit, B., 2005a. Ice in prefermented frozen bread dough - an investigation based on calorimetry and microscopy. Cereal Chemistry 82, 251-255.

Baier-Schenk, A., Handschin, S., von Schönau, M., Bittermann, A.G., Bächi, T., Conde-Petit, B., 2005b. In situ observation of the freezing process in wheat dough by confocal laser scanning microscopy (CLSM): formation of ice and changes in the gluten network. Journal of Cereal Science 42, 255-260. Berglund, P.T., Shelton, D.R., Freeman, T.P., 1991. Frozen bread dough ultrastructure as affected by duration of frozen storage and freeze-thaw cycles. Cereal Chemistry 68, 105-107.

Dieing, T., Hollricher, O., Toporski, J., 2011. Confocal raman microscopy. Springer, Heidelberg. 
Đuričković, I., Claverie, R., Bourson, P., Marchetti, M., Chassot, J.M. and Fontana, M.D., 2011, Waterice phase transition probed by Raman spectroscopy. Journal of Raman Spectroscopy 42, 1408-1412.

Esselink, E. F. J., van Aalst, H., Maliepaard, M., van Duynhoven, J. P. M., 2003, Long-Term Storage Effect in Frozen Dough by Spectroscopy and Microscopy. Cereal Chemistry 80, 396-403.

Fechner, P.M., Wartewig, S., Kiesow, A., Heilmann, A., Kleinebudde, P., Neubert, R.H.H., 2005. Influence of water on molecular and morphological structure of various starches and starch derivatives. Starch/Stärke 57, 605-615.

Jääskeläinen, A.S., Holopainen-Mantila, U., Tamminen, T., Vuorinen, T. , 2013. Endosperm and aleurone cell structure in barley and wheat as studied by optical and Raman microscopy. Journal of Cereal Science 57, 543-550.

Krafft, C., Dietzek, B., Schmitt, M., Popp, J., 2012. Raman and coherent anti-Stokes Raman scattering microspectroscopy for biomedical applications. Journal of Biomedical Optics 17, 040801.

Le Bail, A., Zia, C., Giannou, V., 2012. Quality and safety of frozen bakery products. In: Sun, D.W. (Ed.), Handbook of frozen food processing and packaging, Second edition. CRC Press, Boca Raton, pp. 501528.

Piot, O., Autran, J.C., Manfait, M., 2000. Spatial distribution of protein and phenolic constituents in wheat grain as probed by confocal microspectroscopy. Journal of Cereal Science 32, 57-71.

Piot, O., Autran, J.C., Manfait, M., 2001. Investigation by Confocal Raman Microspectroscopy of the Molecular Factors Responsible for Grain Cohesion in the Triticum aestivum Bread Wheat. Role of the Cell Walls in the Starchy Endosperm. Journal of Cereal Science 34, 191-205.

Piot, O., Autran, J.C., Manfait, M., 2002. Assessment of cereal quality by micro-Raman analysis of the grain molecular composition. Applied Spectroscopy 56, 1132-1138. 
Rösch, P., Harz, M., Peschke K.-D., Ronneberg O., Burkhardt H., Popp, J., 2006. Identification of Single Eucaryotic Cells with Micro-Raman Spectroscopy. Biopolymers 82, 312-316.

Smith, A. E., Zhang, Z., Thomas, C. R., Moxham, K. E., Middelberg, A. P., 2000. The mechanical properties of Saccharomyces cerevisiae. Proceedings of the National Academy of Sciences 97, 98719874.

Smith, E., Dent, G., 2005. Modern Raman spectroscopy: a practical approach. Wiley, Chichester.

Stoddard, F. L., 1999. Survey of starch particle-size distribution in wheat and related species. Cereal Chemistry, 76, 145-149.

Tuma, R., 2005. Raman spectroscopy of proteins: from peptides to large assemblies. Journal of Raman Spectroscopy 36, 307-319.

Thygesen, L.G., Løkke, M.M., Micklander, E., Engelsen, S.B., 2013. Vibrational microspectroscopy of food. Raman vs. FT-IR. Trends in Food Science and Technology 14, 50-57.

Weikusat, C., Freitag, J., Kipfstuhl, S., 2012. Raman spectroscopy of gaseous inclusions in EDML ice core: first results - microbubbles. Journal of Glaciology 58, 761-766.

Yang, D., Ying, Y., 2011. Applications of Raman spectroscopy in agricultural products and food analysis: A review. Applied Spectroscopy Reviews 46, 539-560.

Yi, J., Kerr, W.L., 2009. Combined effects of freezing rate, storage temperature and time on bread dough and baking properties. LWT - Food Science and Technology 42, 1474-1483.

Zhao, Y., Ma, C.Y., Yuen, S.N., Phillips, D.L., 2004. Study of Acetylated Food Proteins by Raman Spectroscopy. Journal of Food Science, 69, 206-213.

Zounis, S., Quail, K.J., Wootton, M., Dickson, M.R., 2002. Effect of Final Dough Temperature on the Microstructure of Frozen Bread Dough. Journal of Cereal Science 36, 135-146. 
331 Table 1: Spectral ranges selected as characteristic for the single dough components. The references

332 cited provide a detailed discussion of the Raman spectra and of the band assignment for the

333 individual dough components.

\begin{tabular}{|l|l|l|l|}
\hline Dough component & Spectral range $\mathbf{( c m}^{-1} \mathbf{)}$ & Band assignment & Reference \\
\hline Ice & $3080-3200$ & OH stretching band & Euričković et al., 2011 \\
\hline Starch & $460-510$ & $\begin{array}{l}\text { Stretching vibration of } \\
\text { the carbon network }\end{array}$ & $\begin{array}{l}\text { Piot et al., 2000 } \\
\text { Fechner et al., 2005 }\end{array}$ \\
\hline Gluten & $1645-1690$ & Amide I (partly) & Piot et al., 2000 \\
\hline Yeast & $740-766$ & $\begin{array}{l}\text { Ring breathing } \\
\text { vibration of } \\
\text { tryptophan (possibly) }\end{array}$ & Rösch et al., 2006 \\
\hline
\end{tabular}

\section{Figure Captions}

Figure 1: Raman spectra of single dough components: ice, liquid water, starch, gluten and yeast. The spectral ranges used for band integration imaging are marked in blue.

Figure 2: First sample: Distribution of the dough components according to both data processing methods (left: band integration; right: multiple regression). Colour code of the bottom image: starch $=$ red, gluten $=$ yellow ice $=$ white, liquid water $=$ green . green.

Figure 4: Third sample: Distribution of the dough components according to both data processing methods. Colour code of the bottom image: $\operatorname{starch}=$ red, gluten $=$ yellow, ice $=$ white, liquid water $=$ green. 
10. Figures

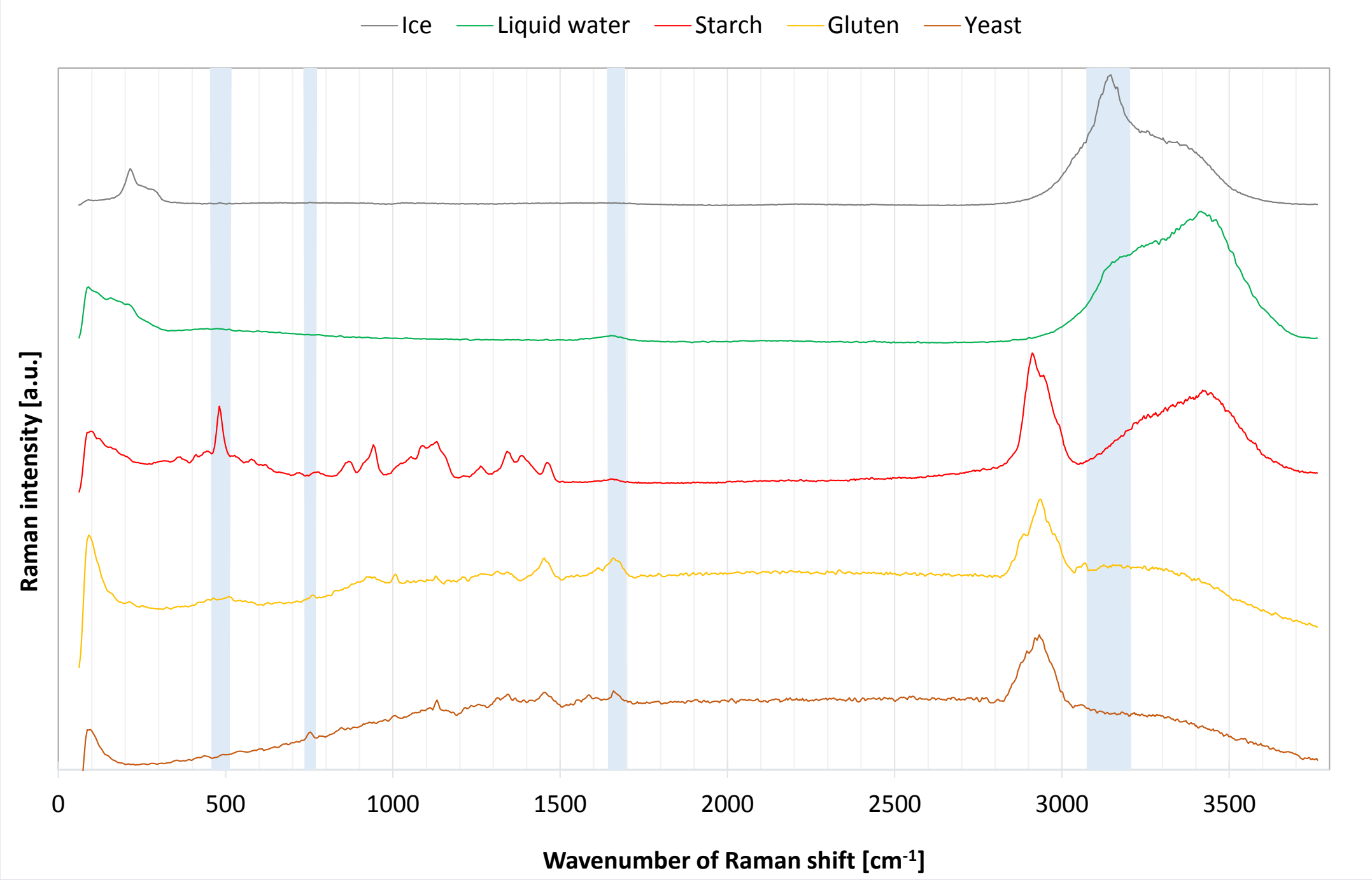

Figure 1 


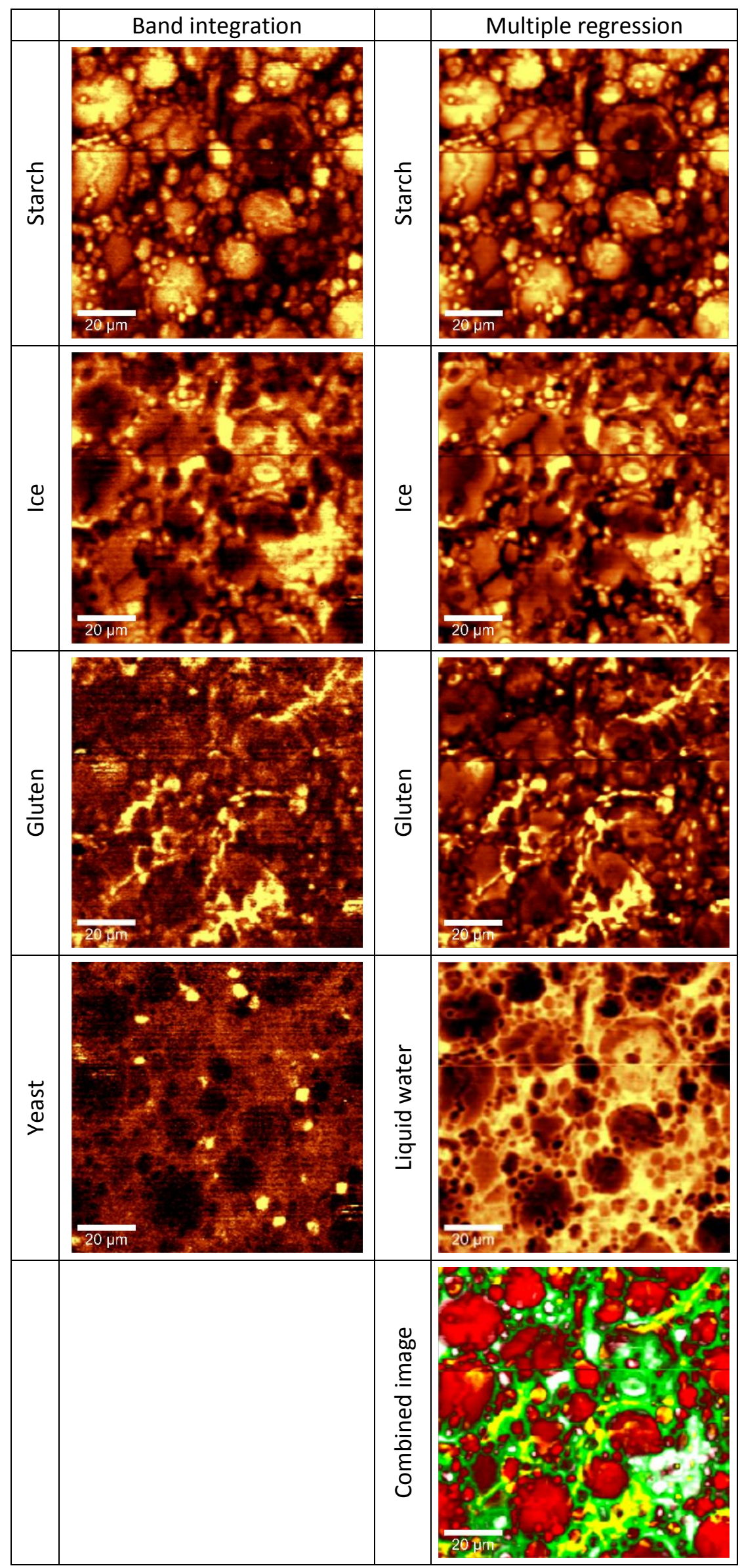

Figure 2 


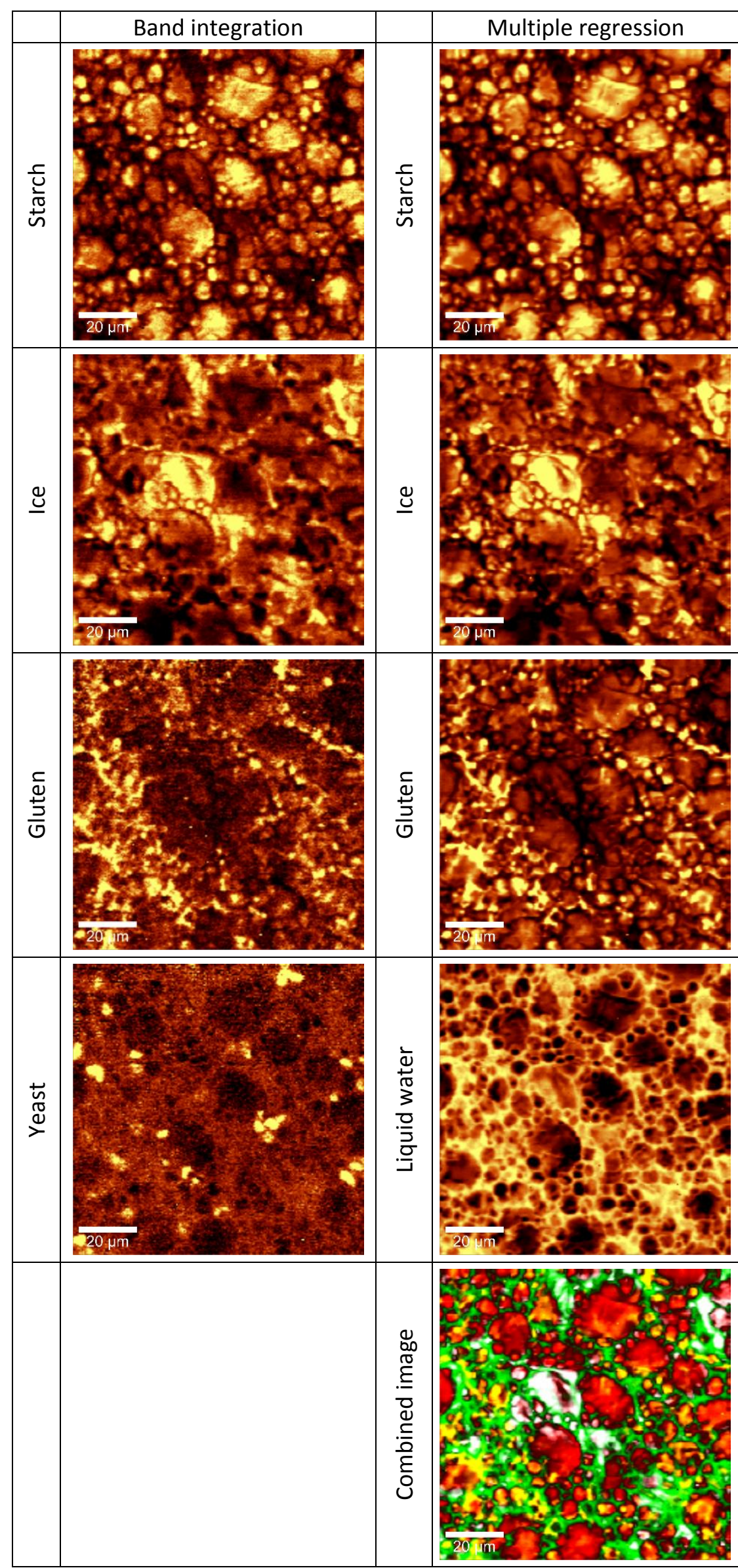

Figure 3 


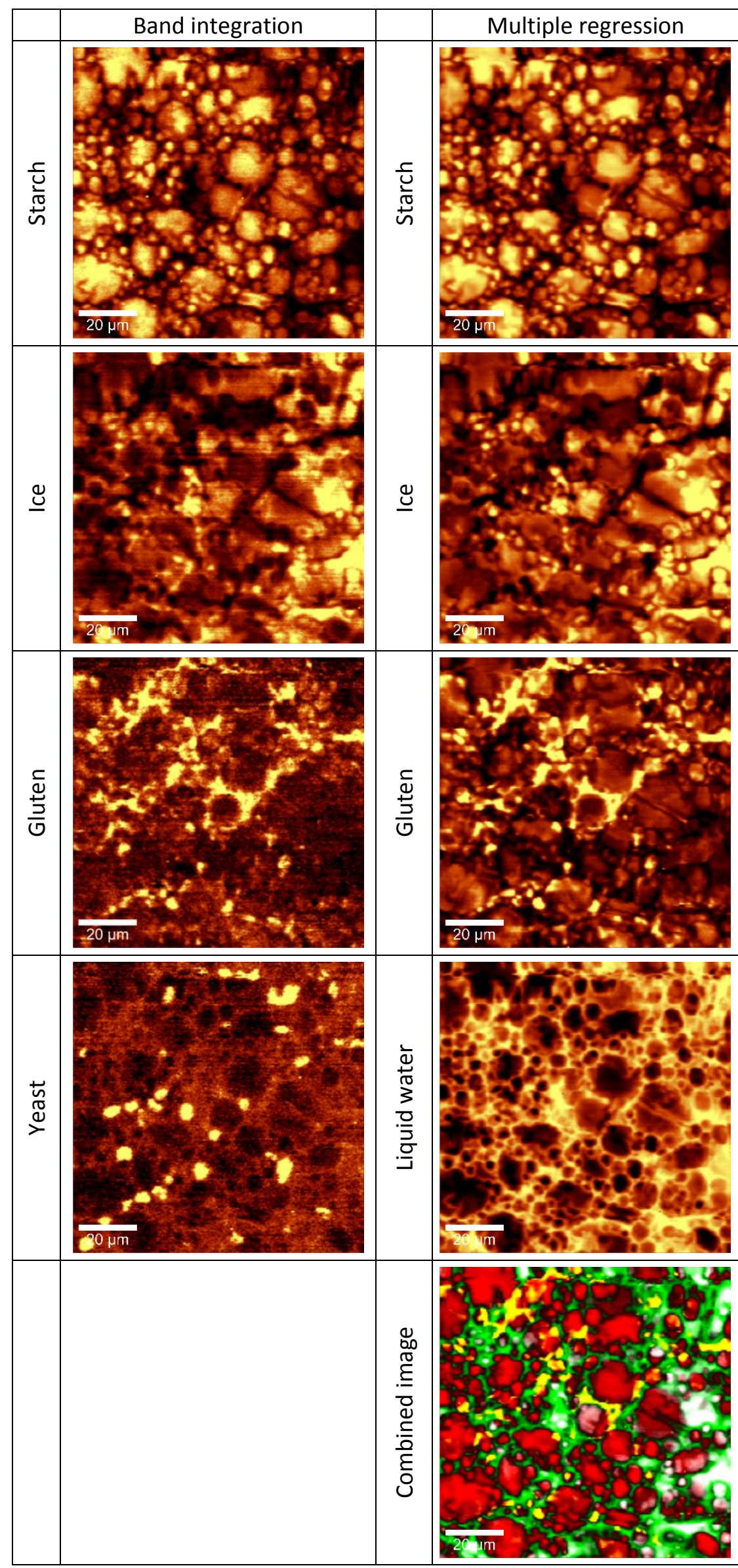

Figure 4 